\title{
Memoria y Saber. Factores asociados a las pérdidas poscosecha en cultivos de Naranja Salustiana Memory and Knowing. Factors associated with losses post-harvest in crops of salustian orange
}

\author{
Juan Esteban Giraldo Bartolo ${ }^{1}$, Rafael Rincón Arango ${ }^{1}$, María Teresa Torres de Castro ${ }^{2}$ \\ juan.281910894@ucaldas.edu.co1,rafael.281910898@ucaldas.edu.co2,maria.torres_d@ucaldas.edu.co3 \\ 1Administración de Empresas Agropecuarias \\ Universidad de Caldas \\ Manizales, Colombia \\ 2Administración de Empresas Agropecuarias \\ Universidad de Caldas \\ Manizales, Colombia
}

\begin{abstract}
Resumen- En esta investigación se propuso identificar y analizar el uso que dan los patrones de corte al material didáctico y la intencionalidad en la ejecución de sus estrategias educativas para que los recolectores de la fruta aprendan significativamente los conceptos de gran interés y aplicación, tanto en la vida diaria como en los procesos biológicos en campo, y así emitir una respuesta satisfactoria para su contexto laboral. La investigación se fundamenta en un paradigma cualitativo, con el uso de técnicas como la observación semiestructurada y la entrevista. Se concluirá que tanto el patrón de corte como recolectores de la fruta conoce de la importancia del manual didáctico; sin embargo, carecen de elementos para llevarlos a la práctica para el uso fundamental en el proceso de aprendizaje significativo que requiere el operario en el uso del material didáctico.
\end{abstract}

\section{Palabras clave: Didáctica, Post cosecha, Recolector.}

Abstract- In this research, it was proposed to identify and analyze the use that cutting patterns give to the teaching material and the intentionality in the execution of their educational strategies so that the fruit pickers learn significantly the concepts of great interest and application both in daily life. as in biological processes in the field and thus issue a satisfactory answer for their work context. The research is based on a qualitative paradigm, with the use of techniques such as semi-structured observation and interviews. It will be concluded that both the cutting pattern and the fruit pickers are aware of the importance of the didactic manual; however, they lack elements to put them into practice for the fundamental use in the meaningful learning process that the operator requires in the use of the didactic material.

\section{Keywords: Didactics, Post harvest, Collector.}

\section{INTRODUCCIÓN}

"La esencia de Naranja, su ADN, se refleja en la alegría de trabajaren equipos, con metas claras y motivadoras para que los Colaboradores puedan facilitar con alegría y compromiso la vida de nuestros clientes"

Alejandro Asrin

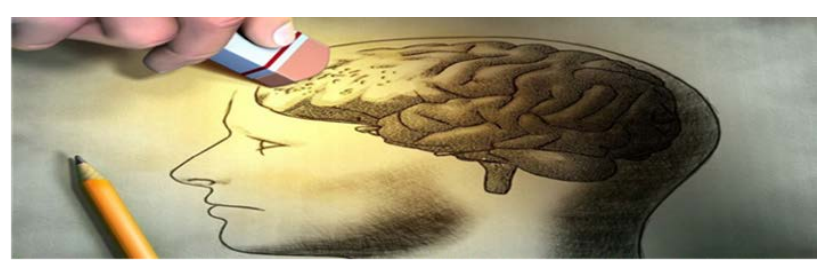

Figura 1: ¿Cómo aprende el ser humano?

Los seres humanos aprendemos de forma empírica, reuniendo y almacenando información según el medio donde nos encontremos, según su curiosidad, su experiencia, vivencias, necesidades y dudas que se presenten y que ponen al servicio de aquellas situaciones que tienden a hacer que otras personas aprendan de ellos.

A lo largo de la historia de la educación hemos puesto poca atención a este proceso. Hoy muchos están de acuerdo en dar prioridad a la inteligencia social, al papel de los sentimientos y la efectividad en el desarrollo de la actividad mental. Esta perspectiva considera que la cualidad característica de la especie humana no es la capacidad de comprender la organización del mundo, sino la constante interpretación del contenido de la mente de los demás, manifestada de diferentes formas: palabras, acciones, producciones.

Tal es el sentido del concepto de comprensión: "Comprender una mente ajena y comprender a sí mismo en el interior de esa capacidad" (García Carrasco, 1.999). De acuerdo con Zsita et al (2012), los cítricos son cultivos permanentes y en general tienen alta adaptabilidad a diversas condiciones climáticas, facilitando su cultivo en un gran número de países, aunque las regiones localizadas por excelencia son el continente americano y europeo. La producción de cítricos lidera el primer lugar en el mundo en relación a otras frutas. Durante el periodo 2006-2007, se registró una producción de aproximadamente 120 millones de toneladas (FAO, 2007). Los cítricos se cultivan comercialmente en más de 50 países. La contribución de la industria citrícola a la economía mundial se estima en más de 10 billones de dólares anualmente (Ladaniya, 2007). 
Basados en esta información, se pretende dar a conocer a la comunidad recolectora de la naranja salustiana un aspecto fundamental a tener en cuenta en el manejo postcosecha de la fruta y es que, ésta continúa viva aún después de cosechada. En tal sentido, la naranja recolectada continúa respirando, madurando en algunos casos e iniciando procesos de senescencia (cambios estructurales), lo cual implica una serie de cambios estructurales, bioquímicos y de componentes que son específicos para la fruta.

Asimismo, el producto obtenido está constantemente expuesto a la pérdida de agua debido a la transpiración y a otros fenómenos fisiológicos.

No obstante, la producción se ve afectada por las altas pérdidas tanto en calidad como en cantidad de nuestros productos en el período de poscosecha debido a factores de orden tecnológico.

La producción, manejo de cosecha, poscosecha, distribución y comercialización, son fundamentalmente los constituyentes básicos del proceso económico en la actividad agrícola. Estas etapas requieren para su aplicación y estudio los suficientes conocimientos técnicos, en el caso de la fruticultura, el manejo de poscosecha, constituye un aspecto crucial para el estímulo y organización de la producción.

Los cítricos, de los cuales hace parte la naranja salustiana, son considerados entre los frutales más importantes en el mundo. Gran parte del fruto se pierde por el escaso conocimiento y pocos recursos por parte de los cultivadores. Existe una amplia gama de agentes fitopatógenos que afectan el cultivo de naranja en sus diferentes etapas vegetativas y reproductivas como son la susceptibilidad del cultivo a plagas, uso indiscriminado de plaguicidas y la falta de tecnología en el manejo.

Cuando hablamos del recolector y su papel importante en los procesos y procedimientos de la participación, considerada según Max Neef como una necesidad humana sentida en conexión con el ser, tener y estar de las personas. (Max-Neef, A, 2.008). Si promovemos la participación, fortalecemos las posibilidades de transformación, tanto a nivel individual como colectivo, generando niveles crecientes de autonomía e independencia para el mejoramiento integral de las condiciones de vida, por ende esta investigación se fundamenta en la metodología participativa para la intervención social porque según Flor Abarca Alpízar, tiene estrecha relación con todos y cada uno de los momentos de los procesos de aprendizaje, garantiza su efectividad, unidad e integralidad para la realización de procesos de interaprendizaje de impacto real en la vida de los recolectores, y se requiere la participación activa de los grupos y actores implicados, para orientar y estimular la práctica estimuladora y el cambio social.

Por las razones expuestas anteriormente, se plantea el siguiente interrogante:

¿De qué manera se puede llevar a cabo el desarrollo de una experiencia de aprendizaje por parte del personal encargado del manejo de los procesos y procedimientos en la poscosecha de la naranja salustiana bajo el enfoque comunicativo?

\section{CONTEXTO}

Durante mucho tiempo se consideró que el aprendizaje era sinónimo de cambio de conducta, esto, porque dominó una perspectiva conductista de la labor educativa; sin embargo, se puede afirmar con certeza, que el aprendizaje humano va más allá de un simple cambio de conducta y conduce a un cambio en el significado de la experiencia.

Refiere, (David Ausubel, 1978) "El aprendizaje significativo, es un proceso intencional y orientado que posibilita establecer vínculos sustantivos y no arbitrarios de los nuevos contenidos que se ha de aprender y aquellos que se encuentran en la estructura cognitiva de sujeto que aprende".

Según Gagné el proceso de aprendizaje no debe considerarse como un proceso de maduración mientras el individuo crece, pues el proceso de aprendizaje se da de una forma persistente modificando la conducta como manifestación de cambio en el tiempo.

En la metodología, el interés de este autor no es el aprendizaje en sí, sino en las condiciones que determina el aprendizaje posterior, teniendo en cuenta las capacidades previas y las condiciones externas del individuo que influyen en el desarrollo de su aprendizaje pasando por varias: a) reacción a una señal, b) estímulo respuesta, c) encadenamiento, d) asociación verbal, e) discriminación múltiple, f) aprendizajes de conceptos, g) aprendizaje de principios, h) resolución de problemas.

Este encadenamiento tiene en cuenta lo que el individuo ha aprendido. La metodología propuesta es que haya un proceso de enseñanza aprendizaje más dinámico teniendo en cuenta cuatro aspectos: actitud de compromiso, creatividad, conocimiento sobre estrategias pedagógicas, didácticas $\mathrm{y}$ prácticas.

Al llegar a la edad adulta el ser humano empieza a presentar cambios de manera progresiva, siendo la capacidad cognitiva una de las principales características en disminuir. Las funciones como memoria, atención, concentración, razonamiento y juicio son claves para la autonomía de las personas al interactuar con el mundo, por lo que las alteraciones de dichas capacidades pueden dificultar su diario vivir.

El objetivo de la investigación es minimizar las pérdidas poscosecha, así como la efectividad y la eficiencia de métodos técnicos que utiliza el patrón de corte en capacitaciones a los recolectores de la fruta, se observa necesario contribuir a una revisión de la metodología empleada en las charlas que posibilite una mejora en la productividad.

Las observaciones que se realizan en los predios donde se cultive la naranja salustiana, servirán como instrumento de ayuda para perfeccionar las condiciones cómo se maneja la fruta en la poscosecha por parte de los patrones de corte y los recolectores. Además, es esencial instruir y comprometer mediante actividades lúdicas al personal involucrado en dicho proceso.

En un inicio, la ciencia fue entendida como una suma de hechos regidos por leyes que puede extraerse directamente si se observan los hechos con una metodología adecuada. Sin embargo, ahora se sabe que la ciencia no es un discurso sobre lo "real "sino de un proceso socialmente definido para la elaboración de modelos que sirven para interpretar la realidad. (Gómez, 2006). 
Con lo anterior podemos decir que el estudio planteado proporcionará alternativas de solución a varios problemas que se presentan cotidianamente en nuestro quehacer diario, por ende, en lo referido a nuestro estudio, como es el resolver los problemas en las actividades poscosecha siendo el caso de la naranja salustiana, a fin de optimizar pérdidas que no benefician a los productores.

Como lo menciona Arias, (2000), los cítricos están constituidos principalmente por 80 a $85 \%$ de sólidos totales; prácticamente no contienen almidón y su contenido de proteinas y grasa es alta. La naranja como fruta no climatérica se debe cosechar pertinentemente para evitar que se afecte su calidad organoléptica durante la fase postcosecha.

De acuerdo con Ceballos (2010), las pérdidas poscosecha en cultivos de cítricos están relacionadas principalmente por la mala manipulación del fruto. Las pérdidas se originan por daños mecánicos, almacenamiento inadecuado, manipulación, transporte incorrecto. Lo anterior, con el fin de que haya reducción de pérdidas de la fruta en la poscosecha y que se aplique el manejo de material didáctico, mejoraría el nivel de producción y la función afectiva y cognitiva de esta población, llevando así a una optimización de los riesgos; ya que las interacciones entre conocimiento, instrucción y uso de estrategias didácticas constituyen una tríada de beneficios.

\section{Objetivo General}

Analizar los factores que inciden en las pérdidas poscosecha de la naranja salustiana y según las falencias encontradas elaborar un manual que sirva de apoyo al recolector para minimizar estos riesgos.

\section{Objetivos Específicos}

$\checkmark$ Determinar los factores bióticos, abióticos y antropológicos de la naranja salustiana.

$\checkmark \quad$ Realizar un sondeo de los conocimientos asociados a los procesos de postcosecha usados por el operario.

$\checkmark$ Interactuar con el operario en relación al manejo racional y factores de riesgo que se pueda presentar con el uso inadecuado de las técnicas en la postcosecha de la naranja salustiana.

$\checkmark$ Elaborar material ilustrativo con actividades didácticas sobre las técnicas adecuadas en los procesos y procedimientos de la poscosecha de la fruta.

\section{DESCRIPCIÓN}

Los materiales didácticos han servido de apoyo en los aprendizajes del ser que se forma, cada uno es seleccionado y empleado dependiendo el propósito que se pretende obtener.

El desarrollo de esta investigación se ha construido de acuerdo al problema de investigación. Daremos definición de reflexión que respalda la investigación, reflexión teórica acerca del aprendizaje y los diferentes conceptos de manejo poscosecha de la naranja salustiana.

\section{Reflexiones que respaldan la investigación:}

Podemos citar a la investigación de Quenta, (2013): Incidencia de los métodos activos en el aprendizaje significativo de los sujetos que aprenden; donde el investigador hace mención sobre el poco uso de los orientadores sobre los métodos activos para lograr aprendizajes significativos durante el proceso de enseñanza - aprendizaje, el mismo que permite la participación activa de las personas en la construcción de sus propios aprendizajes, despertando al mismo tiempo en ellos la curiosidad por la investigación, poniendo de manifiesto sus aptitudes físicas y mentales, despertando en ellos el interés y la curiosidad por aprender; siendo un reto para el dirigente el pensar y crear situaciones de aprendizaje altamente interesantes, estimulantes y significativas.

\section{Reflexiones teóricas acerca del Aprendizaje:}

La Organización para la Cooperación y el Desarrollo Económico, (OCDE, 2012) considera que actualmente el concepto que impera es el socio constructivista, en donde se entiende que el aprendizaje se da por la situación del contexto con una construcción muy activa mediante la negociación con los pares, con la sociedad; siendo el fin último la adquisición de habilidades adaptativas, dichas habilidades se deben aplicar en las diferentes situaciones por las que se vea inmerso el ser humano, en donde le permita salir airoso y realmente lo que aprendió le sirva para la vida, transformando positivamente su entorno y el de los demás.

Mientras que, para Ballester, (2002), el aprendizaje es la construcción del conocimiento en donde unas piezas encajan con las otras convirtiéndose en un todo coherente, produciéndose de esta manera un auténtico aprendizaje, un aprendizaje a largo tiempo que no será sometido al olvido. Para que esto suceda y se genere un aprendizaje a largo plazo, es necesario conectar una buena estrategia didáctica por parte del docente con las ideas previas y la experiencia que el estudiante ya posee, donde se van construyendo las ideas interconectadas unas con otras $y$ formando la red del conocimiento.

Aunque es cierto que los adultos aprenden de forma diferente, ello no significa que lo hagan con mayores dificultades, sino que éstas son propias y particulares de este grupo. De hecho, la base de su aprendizaje debe ser reflexivo, introspectivo y experiencial. En relación a la investigación, la memoria y el saber que impera en el productor y su relación con las pérdidas en la poscosecha de la naranja matiza su conocimiento, vivencias, emociones y experiencias con el reto de diseñar estrategias educativas que cumplan con las habilidades del adulto y le permitan alcanzar sus metas, las cuales suelen estar ligadas a su vida diaria y ambiente laboral, de manera efectiva e inmediata.

\section{Conceptos Referenciales a la investigación: Estrategia didáctica:}

Acciones planificadas por el orientador con el objetivo de que el que aprende logre la construcción del aprendizaje y se alcancen los objetivos planteados. Una estrategia didáctica es, en un sentido estricto, un procedimiento organizado, formalizado y orientado a la obtención de una meta claramente establecida. Su aplicación en la práctica diaria requiere del perfeccionamiento de procedimientos y de técnicas cuya elección detallada y diseño son responsabilidad del que enseña.

Naranja: Es un alimento del reino vegetal dentro de la dieta del hombre, de la familia rutácea, género citrus y especie Sinensis. En lo que se refiere al tipo de alimento, pertenece al 
grupo frutas, y por sus características lo enmarcamos dentro de la rama de cítricos.

Cosecha: Es el momento adecuado para la recolección y permitir que esta llegue en buenas condiciones al mercado sin sufrir daños o pérdida de calidad.

Manejo post cosecha: Este manejo se ha convertido en una habilidad que utiliza muchos sistemas tecnificados con el propósito de reducir pérdidas, vigilar calidades y garantizar buenas prácticas de manejo sanitario. Según Báez et al (2014) el mal manejo post cosecha es un problema que afecta la economía de productores, comercializadores y consumidores. Las pérdidas post cosecha de la naranja pueden lograr de 10 a $25 \%$, del volumen producido, dependiendo de la zona, variedad y prácticas de cultivo.

Pérdidas en el manejo post cosecha de la naranja: Después de la cosecha, según Fonseca (2007), los cítricos evolucionan hacia la senescencia pérdida gradual de calidad comercial por distintos motivos los cuales son: pérdida de peso y textura por deshidratación, ablandamiento del fruto, deformación, envejecimiento de la cáscara, incremento de alteraciones fisiológicas, podredumbres, aumento en el índice de madurez por disminución del contenido de ácido cítrico, pérdidas de sabor y aroma, reducción del contenido de vitamina $\mathrm{C}$, disminución del valor alimenticio.

El mal manejo post cosecha es un problema que afecta la economía de los productores, de los comercializadores y los consumidores. En el manejo de la post cosecha puede darse pérdidas en la selección, limpieza y clasificación.

Las estrategias didácticas utilizadas de acuerdo a la preferencia del investigador para el aprendizaje significativo de los recolectores fueron: dominó, parques, bingo, láminas de la poscosecha de la naranja mostrando los factores bióticos, abióticos y antropológicos de la fruta que se ven afectadas por la acción de la manipulación. Se utilizan para ayudar a comunicar una idea que el usuario necesite aprender o reflexionar de forma clara y que capte el concepto, hacer lo propio, relacionarlo con su entorno, relacionarlo con su labor en campo y propiciando el proceso de aprendizaje.

El aprendizaje es el proceso por el cual se adquieren o modifican habilidades, destrezas, conocimientos, conductas o valores como resultado del estudio, la experiencia, instrucción, razonamiento y observación, es el proceso mediante el cual se adquiere una habilidad, se asimila una información o se adopta una nueva estrategia de conocimiento y acción por esta razón debe ser significativo.

Por esta razón Ausubel como precursor del aprendizaje significativo afirma que: "El aprendizaje significativo propone tanto que el alumno manifiesta una actitud hacia el aprendizaje significativo; es decir, una disposición para relacionar no arbitraria sino sustancialmente, el material nuevo con su estructura cognoscitiva.

Estudio de Caso: se puede definir como el examen de un ejemplo en acción, es decir, el estudio de unos hechos específicos (el caso: relación memoria y saber) y la recogida selectiva de información relacionada con esos hechos específicos con el objetivo de obtener conocimiento de esa experiencia.

La investigación tiene un enfoque cualitativo y de alcance descriptivo, pues busca conocer las vivencias y actitudes que se presentan, primero con el recolector en relación con el conocimiento y uso que tiene sobre la interacción con la fruta en relación con los riesgos ergonómicos en la recolección y segundo el compromiso frente al material didáctico, también es posible afirmar que es propositiva porque fue un proceso que buscó generar una recopilación de actividades lúdicas partiendo de las vivencias de los participantes.

La población estudio fueron los recolectores de la fruta. Adicionalmente se realizaron entrevistas a profundidad, las que se han considerado como el medio clave para un dialogo abierto. La técnica aplicada fue la entrevista a profundidad. Las preguntas no fueron predeterminadas puesto que el objetivo era motivar la conversación del participante y del investigador, explorando creencias, vivencias pensamientos y sentimientos alrededor de las temáticas abordadas.

Las entrevistas se basaron principalmente en las siguientes categorías de análisis:

1) Hablando sobre salud de los cítricos, 2) Importancia y necesidad del manual ilustrativo en la fase poscosecha como estrategia pedagógica, 3) Estructura, composición, fisiología y bioquímica de la naranja salustiana, 4) Fundamento en los procesos de manipulación y herramientas en la poscosecha, 5) Efecto de la temperatura. Pérdida de agua y humedad. Almacenamiento en atmósferas controladas, 6) Alteraciones fisiológicas y patológicas de la naranja salustiana.

En el cuestionario se formularon preguntas en las que se hace una introducción previa, instrucciones específicas y datos usuales; para la elaboración de las preguntas se tiene en cuenta el conocimiento general acerca de los factores bióticos, factores abióticos y antropológicos.

Basados en cada encuentro y en el conocimiento de los recolectores, se realizó un manual que se fue construyendo cada semana con las actividades programadas para cada sesión, para que así, se cumpliera con el objetivo planteado.

Tabla 1. Instrumentos de Apoyo

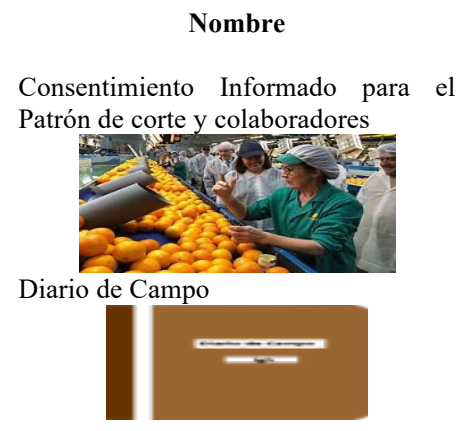

Cuestionario para el Patrón de Corte

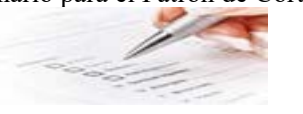

Cuestionario para los colaboradores de corte

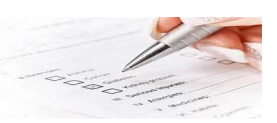

\section{Aplicación}

Busca dar herramientas en la información para facilitar la toma de decisiones en la participación del estudio con un riesgo mínimo.

Herramienta que permite sistematizar las experiencias para luego analizar los resultados.

Pre diagnóstico sobre la conservación y la calidad final de los cítricos e importancia de la implementación del manual didáctico en la poscosecha.

Pre diagnóstico. Implementación de estrategias educativas en los procesos biológicos y químicos de la naranja salustiana en la fase de la poscosecha. 


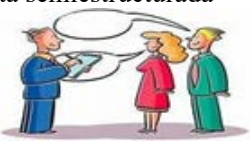

Las Preguntas no son predeterminadas puesto que el Objetivo es motivar la conversación del participante y del investigador, explorando creencias, vivencias, pensamiento y sentimientos alrededor de las temáticas abordadas

\section{Resultados}

En el cuadro que se presenta a continuación, se hace la presentación de los resultados obtenidos en las entrevistas y talleres que se realizaron al personal encargado de la poscosecha de la naranja con los que se llevó a cabo la investigación, en la cual se contempla las categorías y subcategorías y fuentes que sirvieron de referencia en el proceso, así como los testimonios posteriormente el análisis interpretativo.

Tabla 2. Datos obtenidos según aplicación de los instrumentos

Categorías

Subcategorías

Datos a Observar

\section{RECURSOS DIDÁCTICOS}

ESTRATEGIAS DIDÁCTICAS

$\begin{array}{ll}\text { TECNICAS DE } & \text { Trabajo Colaborativo } \\ \text { ENSEÑANZA } & \text { Creatividad } \\ & \begin{array}{l}\text { Disponibilidad frente al } \\ \text { aprendizaje. }\end{array}\end{array}$

PARTICIPANTES Personal recolector de la naranja salustiana

Se encontró que los recolectores de naranja en su gran mayoría presentan desconocimiento sobre los aspectos generales de la manipulación del producto, también muestran disminución moderada de las habilidades cognitivas y mencionan que conservan habilidades sicosociales que les permite realizar ocupaciones de manera independiente.

Para demostrar lo anterior se desarrollaron los siguientes talleres:

Taller 1: Hablando sobre salud de los cítricos: el taller tenía como objetivo reconocer el significado de la salud de la naranja y los factores que la afectan. Comprender que para estar bien el fruto no necesariamente los mecanismos de fumigación son la solución. El material utilizado es el bingo, se ejercitó la memoria haciéndoles preguntas sobre lo que acababa de suceder en el juego. Se encontró que durante el desarrollo de la actividad es necesario mantener el refuerzo verbal para la continuidad del ejercicio, ya que, si no se dan las instrucciones, los participantes se desaniman con prontitud, o perdían el ritmo del juego.

Taller 2: Importancia y necesidad del material ilustrativo en la fase poscosecha como estrategia pedagógica: este taller se orientó a potencializar las habilidades sociales, cognitivas y motrices, mediante diversos juegos como el dominó, lotería y parques. Estos recursos mostraron ser motivadores para los participantes. Sin embargo, se requiere la intervención del investigador para que genere confianza y dar prioridad a la inteligencia social al papel de los sentimientos y la efectividad en el desarrollo de la actividad mental y se pueda disminuir el número de individuos con poco conocimiento en la manipulación de la naranja en la poscosecha.

Taller 3. Estructura, composición, fisiología y bioquímica de la naranja salustiana: La meta de este taller fue conocer la importancia del buen uso de las estrategias didácticas para la efectividad del manejo estructural y funcional de la naranja y la disminución a los factores asociados a la perdida poscosecha. La integración se llevó mediante varios juegos como láminas de los factores bióticos, abióticos y antropológicos mostrando las estructuras que se ven afectadas por acción de efectos contrarios de manipulación donde el recolector manifiesta: "No sabía que la naranja después de arrancada del árbol seguía con vida", "como aquí el patrón de corte no nos informa, por eso no tenemos ese conocimiento".

En el cuadro que se presenta a continuación, se hace la presentación de los resultados obtenidos en las entrevistas y talleres que se realizaron a los recolectores de naranja con los que se llevó a cabo la investigación en el cual se contempla las categorías, subcategorías y fuente que sirvieron de referencia en el proceso, así como los testimonios más relevantes que permitieron realizar posteriormente el análisis interpretativo.

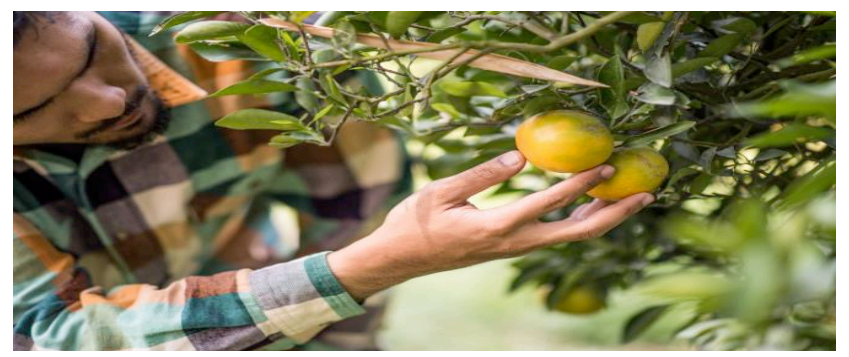

Tabla 3. Preguntas al recolector
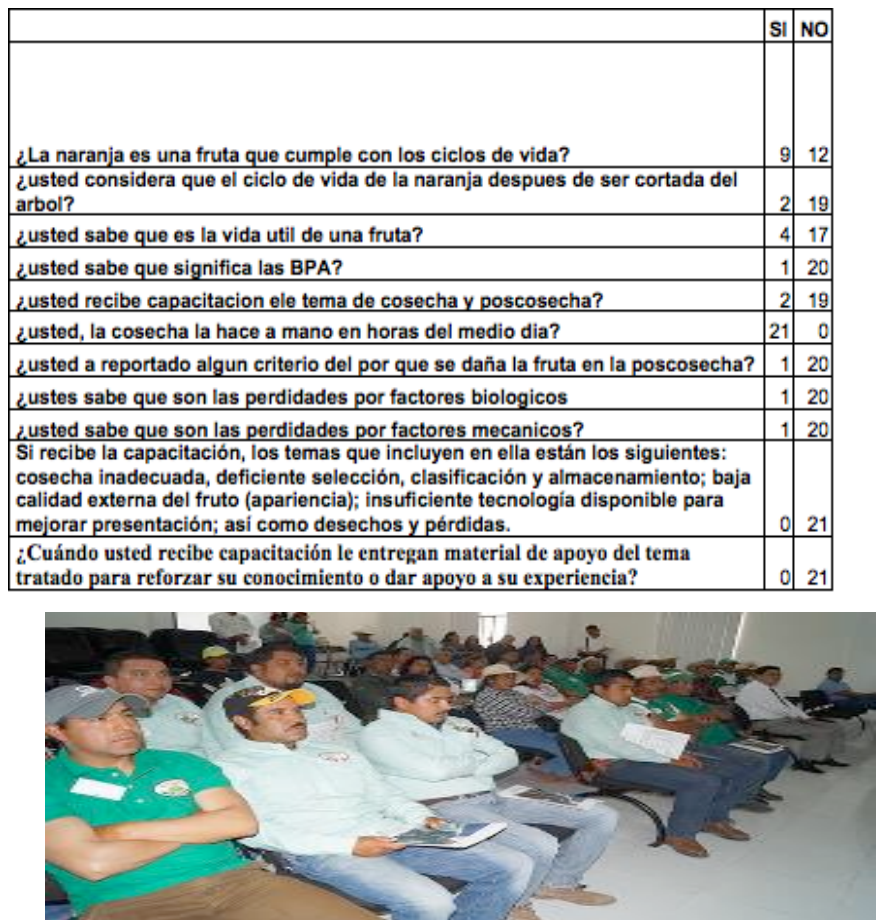
En general se observó que los recolectores poseen poco conocimiento sobre el proceso de cosecha y poscosecha de la naranja, según los resultados del cuestionario el $80 \%$ del re colector son personas que, por factores sociales como el desplazamiento, la pobreza o porque desde muy temprana edad se vieron avocados a conseguir el sustento. Mediante el análisis general de los resultados obtenidos en la práctica de los talleres se identifica que es necesario y beneficioso realizar diferentes tipos de encuentros que involucren el ejercicio de estimulación emocional y cognitiva y su valor como seres sociales.

\section{CONCLUSIONES}

Con las estrategias planteadas para desarrollar la investigación, se puede decir que el material didáctico es un factor determinante en el proceso de aprendizaje significativo para el personal recolector de la naranja salustiana, de forma positiva atractiva y motivante que les va a permitir generar información pertinente a cerca del por qué deben acudir al manejo de estrategias pedagógicas, además, se hace notorio que la memorización no es suficiente al impartir una charla con procedimientos tradicionales, en este momento es donde se utilizarían actividades lúdicas para atraer la atención debido a que se despiertan sensaciones como la visual, olfativa, auditiva $y$ tangible. Es relevante mencionar que las comunidades de recolectores deben superar las brechas del mal manejo biológico y antropológico del proceso poscosecha de la naranja, para poder hacer frente a las diversas circunstancias que trae una mala manipulación de la fruta.

\section{REFERENCIAS}

García Carrasco, J. (marzo, 2009). Las formas de la alfabetización cultural en la sociedad de la información. Teoría de la Educación. Educación y Cultura en la Sociedad de la Información. (pp. 49-75, vol. 10, núm. 1). Universidad de Salamanca. España. https://www.redalyc.org/pdf/2010/201018023004.pdf.

Millind, L. (2007). Biology, Technology and Evaluation.https://www.amazon.com.mx/Citrus-FruitBiology-Technology-Evaluation/dp/0123741300

Max-Neef, A. (2.008). Una teoría de las necesidades humanas para el desarrollo. http://biblioteca.esucomex.cl/RCA/Una $\% 20$ teor $\% \mathrm{C3} \% \mathrm{~A}$ $\mathrm{Da} \% 20 \mathrm{de} \% 201 \mathrm{las} \% 20$ necesidades\%20humanas\%20para $\% 20 \mathrm{el} \% 20$ desarrollo.pdf.

Ausbel, David. (2.004). Teoría del Aprendizaje Significativo. España, Pamplona. http://cmc.ihmc.us/papers/cmc2004290.pdf.

Gómez, N. A. (2.001). Un mundo de la Metodología de la investigación. PDF. Universidad Colegio Mayor de Nuestra del Rosario. http://www.academia.edu/11232932/Introducci\%C3\%B3 $\mathrm{n}$ a la Metodolog $\% \mathrm{C} 3 \% \mathrm{ADa}$ de la Investigaci $\% \mathrm{C} 3 \%$ B33 - _Cient $\%$ ADfica_Autor_Prof_Marcelo_G\%C3\%B3 mez_1_ 